\title{
RAPID VARIABILITY OF H $\alpha$ EMISSION LINE IN Be STARS
}

\author{
B. G. ANANDARAO and A. CHAKRABORTY \\ Physical Research Laboratory, Ahmedabad - 380009, India \\ and
R. SWAMINATHAN and B. LOKANADHAM Osmania University, Hyderabad - 500007, India

We have initiated an observational campaign on some bright Be stars in order to investigate the rapid variability in emission lines using a FabryPerot spectrometer $\left(\lambda / \delta \lambda=10^{4} ; F S R=21.3 \AA\right)$ at the Nasmyth focus of the $1.22 \mathrm{~m}$ JRO telescope at Hyderabad, India. The PMT dark counts were $1-2 \sec ^{-1}$. Here we report our first observations on four stars.

\section{1. $\gamma$ Cassiopieae}

Within the observation time of about 1 hour on $27 / 12 / 92$, the $H \alpha$ line profile from this star has undergone variability from a well-defined asymmetric shape(Fig. 1a) into disappearance and reappearance sequences with the changes occurring in a few minutes. On 29/12/92, the profile was broader and more spiky and probably there was continuum variation within the $\operatorname{scan} \operatorname{time}(\approx 1.5 \min )$.

\section{2. $\lambda$ Eridani}

The $H \alpha$ emission profiles observed on 29/12/92 show a strong triple- spiked structure(Fig. 1b) which disappeared in the very next scan taken 2 minutes later. On 14/02/93 the $H \alpha$ emission was substantially weaker and broader than in Dec '92. The transient-like episodes were not observed in Feb '93.

\section{3. $\kappa$ Draconis}

This star exhibited rapid variability in the $H \alpha$ emission profile structure on time scales of $\leq 2 \min$ on $14 / 02 / 93$ as well as on $15 / 02 / 93$. The coadded and averaged profile on 14/02/93 shows a double-humped structure with the $V$ component narrower than the $R$ (Fig. 2). The $F W H M$ of the $V$ component is close to the stellar vsini indicating that the shell is in corotation while the $R$ component could be due to earlier ejections circularised after the redistribution of the angular momentum(Hanuschik et al 1993). The absence of these features 24 hours later shows that the corotating and out-going shell has attained circularisation phase during the rotation period viz. 0.8 day, becoming broader and falling back on to the star. 

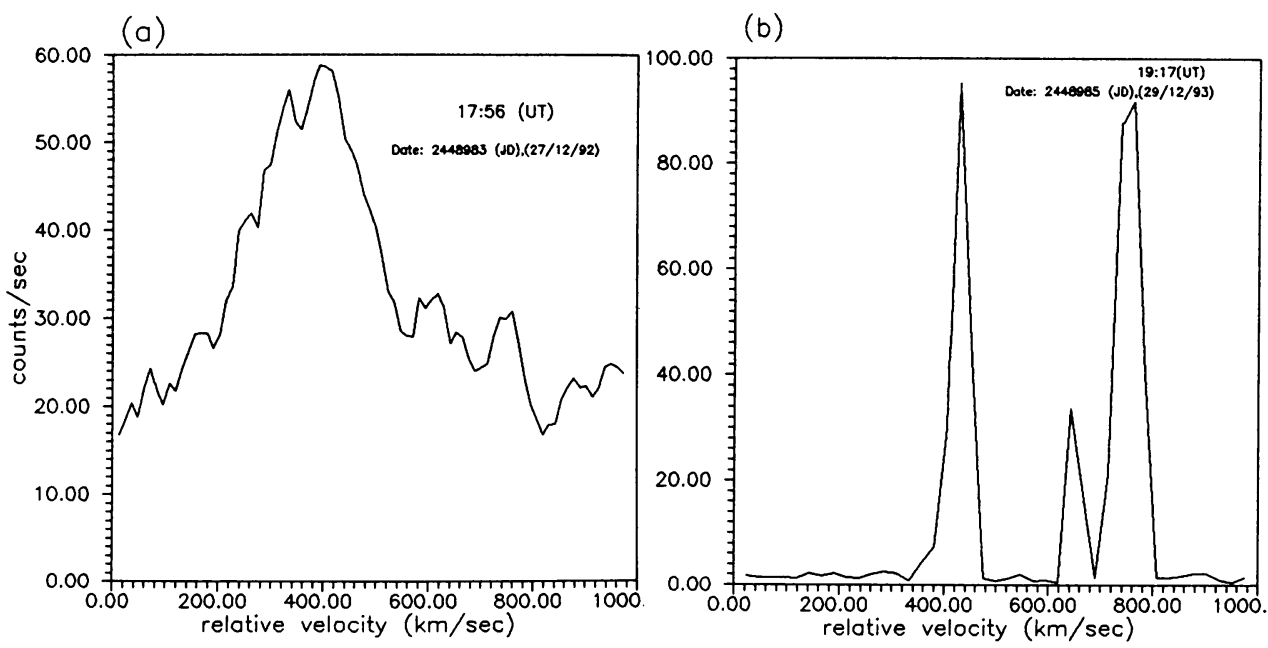

Fig. 1. Profiles of $H \alpha$ emission line from $\gamma \operatorname{Cas}(\mathrm{a})$ and from $\lambda \operatorname{Eri}(\mathrm{b})$

\section{27 Canis Majoris}

Essentially this star showed a similar behavior as $\kappa D r a$ with a doublehumped profiles on both the nights(14 \& 15 Feb '93)(Fig. 3). The $V$ component is corotating with the star and the $R$ component is broader. The presence of the narrower $V$ component on the second night could be attributed to the longer rotation period for $27 C M a(\mathrm{cf} . \kappa \mathrm{Dra})$ and perhaps the circularisation process was not completed and/or there had been fresh ejecta. The presence of broad $\mathrm{HeI}$ emission line indicated that it is associated with the thermalised region. The minute-scale variations are present in this case also.

\section{Conclusions}

Clearly we need to establish the nature of the minute-scale variability in all these stars. These rapid variations cannot be due to radial pulsations and need either a binary hypothesis or flares due to localised magnetic fields(Smith 1991). The day-to-day variability observed in the unique doublehumped emission line profile in $\kappa D r a$ and $27 C M a$ may be understood in terms of episodic mass ejection followed by redistribution of angular momentum.

\section{References}

D. Baade, Vol.36, 59 .

Hanuschik, R.W. et al, 1993, A\&A, 274, 356.

Smith, M.A., 1991, in ESO Workshop on Rapid Variability of OB stars, Ed. 


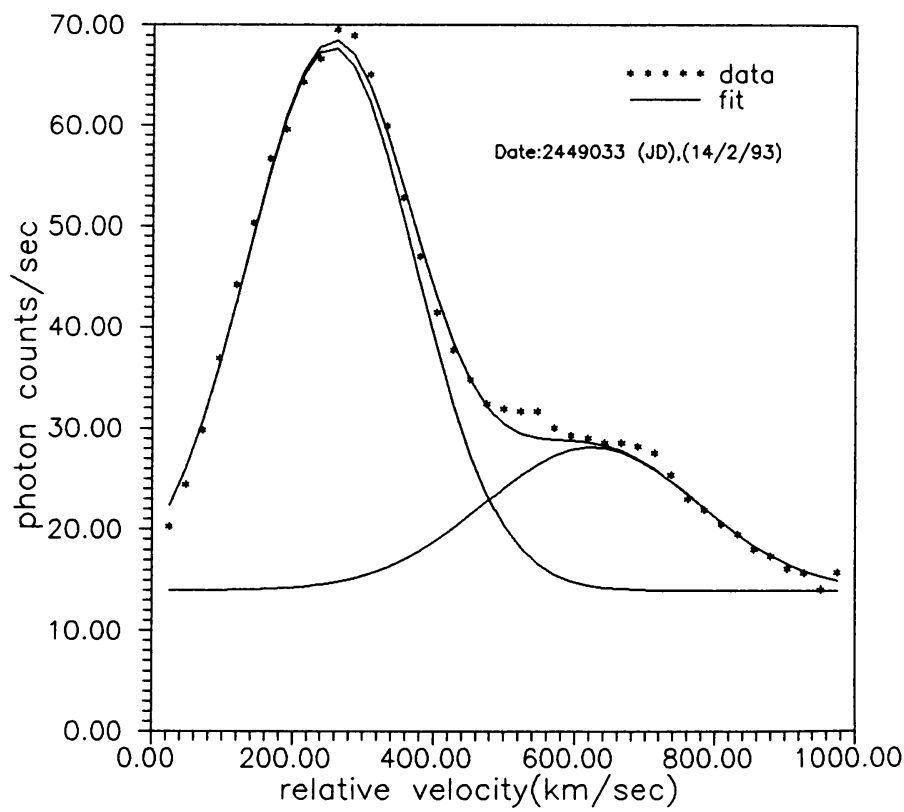

Fig. 2. Profile of $H \alpha$ emission line(36 profiles coadded and averaged) from $\kappa D r a$ on 14 Feb 1993

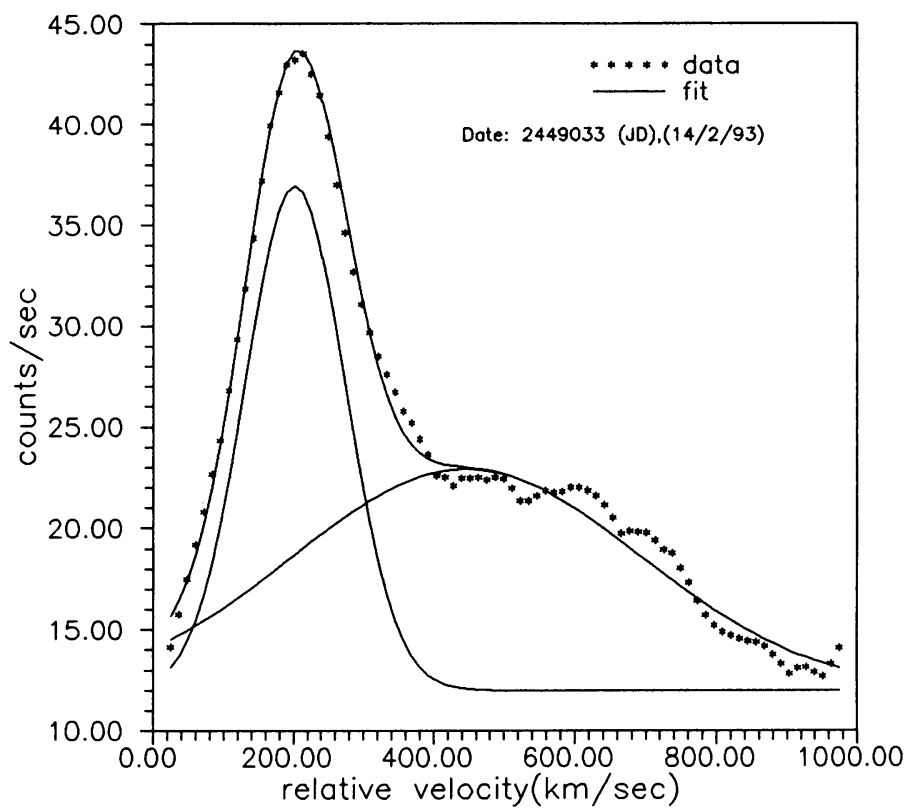

Fig. 3. Profile of $H \alpha$ emission line(36 profiles coadded and averaged) from $27 C M a$ on 14 Feb 1993 